\title{
Cardiovascular Outcomes of Antidiabetes Medications by Race/Ethnicity: a systematic review and meta-analysis
}

Xiaoling Cai ( $\nabla$ dr_junel@sina.com)

Peking University People's Hospital https://orcid.org/0000-0002-7881-0543

\section{Sam Dagogo-Jack}

University of Tennessee University Medical Group

Chu Lin

Peking University People's Hospital

Wenjia Yang

Peking University People's Hospital

Linong Ji ( $\nabla$ jiln@bjmu.edu.cn )

Peking University People's Hospital

Original investigation

Keywords: cardiovascular outcome, race, ethnicity, antidiabetes medication, type 2 diabetes

Posted Date: August 19th, 2020

DOl: https://doi.org/10.21203/rs.3.rs-46807/v1

License: (c) (i) This work is licensed under a Creative Commons Attribution 4.0 International License. Read Full License

Version of Record: A version of this preprint was published at Journal of Diabetes and its Complications on June 1st, 2021. See the published version at https://doi.org/10.1016/j.jdiacomp.2021.107980. 


\section{Abstract}

Background: The consistency of cardiovascular risk reduction by antidiabetes medications across racial and ethnic groups remains unclear. The aim of this study was to analyze racial/ethnic patterns in the results of cardiovascular outcomes trials of antidiabetes medications in people with type 2 diabetes.

Method: PubMed and Cochrane library databases were searched from the inception dates to December 2019. Cardiovascular outcome trials in type 2 diabetes that randomized participants to active or control treatment and reported results by race/ethnic groups or region were included.

Results: A total of 16 studies were included in this meta-analysis. Among White participants, active antidiabetes medication, compared with control treatment, significantly decreased the composite cardiovascular outcomes $(\mathrm{OR}=0.90,95 \% \mathrm{Cl} 0.86-0.94, \mathrm{p}<0.05)$. Among Asian participants, active antidiabetes medication, compared with control treatment, also significantly decreased the composite cardiovascular outcomes $(\mathrm{OR}=0.82,95 \% \mathrm{Cl} 0.76-0.90, \mathrm{p}<0.05)$. Among Black participants $(\mathrm{OR}=0.96,95 \%$ $\mathrm{Cl}$ 0.69-1.32, $\mathrm{p}=0.79)$ and subjects from other groups (mostly Hispanics or Pacific Islanders) $(\mathrm{OR}=0.92$, $95 \% \mathrm{Cl}$ 0.81-1.04, $\mathrm{p}=0.16$ ), active anti-diabetes medication resulted in nominal but non-significant decreases in the composite cardiovascular outcomes, compared with the control treatment.

Conclusions: Antidiabetes drugs demonstrated cardiovascular safety in people with type 2 diabetes from all racial/ethnic groups studied, but achieved significant composite cardiovascular risk reduction only in White and Asian participants, perhaps due to differences in sample size and power.

\section{Research In Context}

\section{Evidence before this study}

Multiple cardiovascular outcomes trials (CVOTs) of antidiabetes medications have been completed and the results show variability among classes of glucose-lowering agents in their effects on cardiovascular events. The disparities of cardiovascular risk reduction by antidiabetes medications across racial and ethnic groups remains unclear.

\section{Added value of this study}

Antidiabetes medications demonstrated cardiovascular safety in people with type 2 diabetes from all racial/ethnic groups studied but achieved significant composite cardiovascular risk reduction only in White participants and Asian participants, but not in Black participants and subjects from other groups (mostly Hispanics or Pacific Islanders), perhaps due to differences in sample size and power.

\section{Implications of all the avaialbe evidence}


Significant cardiovascular benefits by the assessed antidiabetes medications were only confimed in White and Asian population. Given the disproportionate burden of type 2 diabetes and its complications in African Americans, Latinos, Native Americans and other ethnic groups, it is imperative to demonstrate the efficacy of medications and interventions for cardiovascular risk reduction in these populations. The underrepresentation of Blacks and other high-risk groups in the populations enrolled in CVOTs opens the question as whether the benefits demonstrated for Whites and Asians in CVOTs can be also expected to apply to other groups.

\section{Introduction}

Racial and ethnic disparities in the risk factors, prevalence and complications of type 2 diabetes mellitus (T2DM) have been widely reported (1-5). For example, the National Health and Nutrition Examination Survey (NHANES) estimates show approximately 2-fold or higher prevalence of diagnosed T2DM in African Americans, Hispanic Americans, Asian Americans and American Indians, compared with nonHispanic white Americans $(1,6,7)$. Other reports have indicated that T2DM tends to develop at a younger age and lower body mass index in Asian compared with White or African populations $(8,9)$.

The quality of glycemic control and clinical consequences of the microvascular complications of diabetes, such as retinopathy, amputation, and kidney disease, have also been reported to show ethnic/racial disparities $(4,10,11)$. The disparities in microvascular complications can been eliminated with equal access to care and optimal glycemic control $(12,13)$.

Unlike microvascular complications, which can be prevented by optimal glycemic control, the approach to the prevention of cardiovascular disease (CVD) requires targeting all modifiable cardiovascular risk factors along with glucose control $(14,15)$. Analysis of data from 1,344,899 patients in an insured population followed from 2002 to 2012 showed that Blacks, Latinos, and Asians had lower risk of coronary heart disease (CHD) across all risk categories, compared with Whites (16). However, despite having a lower prevalence of $\mathrm{CHD}$ and less atherogenic lipid profile, Blacks have a higher CHD mortality rate compared with Whites (17-19). The age-adjusted CHD death rates per 100000 were 132.3 for nonHispanic White men versus 146.5 for non-Hispanic Black men, and 67.9 for non-Hispanic White women versus 85.4 for non-Hispanic Black women (18).

Landmark randomized, controlled clinical trials of aggressive glycemic control failed to demonstrate reduction in CVD events in people with T2DM, during the active follow-up period (20-22). However, other more recent studies have tested specific antidiabetes medications (rather than glucose control, per se) for the pre-specified primary outcome of major adverse cardiovascular events (MACE). Several of these cardiovascular outcomes trials (CVOTs) of antidiabetes medications have been completed and the results show variability among classes of glucose-lowering agents in their effects on CVD events. Furthermore, enrollment of subjects into the CVOTs does not appear to reflect any attempt at ensuring racial/ethnic balance in the study populations. Consequently, the data from the individual CVOTs do not provide full insight into the efficacy of the study medications across racial/ethnic groups. Given the 
potential for interplay among prevalent CVD risk factors, genetic background, socioeconomic factors, and other variables, in determining outcomes of the CVOTs in different racial/ethnic groups, we conducted a meta-analysis to explore consistency of results from CVOTs across race/ethnicity.

\section{Methods}

\section{Data Sources and Searches}

This study was conducted according to standard guidelines for conducting and reporting systematic reviews (PRISMA checklist). We searched PubMed and the Cochrane Central Register of Controlled Trials (CENTRAL) for randomized control trials (RCTs) from inception until December 2019.

\section{Study Selection}

The inclusion criteria were: (1) type 2 diabetes cardiovascular outcome trials; (2) prespecified cardiovascular events in the active treatment and control groups were reported by race/ethnicity or region of participants; (3) the incidence rates of prespecified cardiovascular events were reported by race/ethnicity or region of participants, so that it was possible to calculate the cardiovascular events from the rates and the total number of participants. The exclusion criteria were: (1) studies that were not RCTs; (2) non-cardiovascular outcome trials; (3) studies that enrolled participants with type 1 diabetes; (4) trials without report of subgroup analysis stratified by race; (5) trials that did not report MACE in subgroups stratified by race.

The search terms used for this meta-analysis were cardiovascular outcome trials; randomized controlled trials; type 2 diabetes; renal outcomes; placebo-controlled trials.

\section{Data Extraction and Quality Assessment}

We used standardized forms for data extraction. Two authors assessed search results independently and extracted data, focusing on year of journal publication; treatments and baseline demographic and clinical characteristics; and cardiovascular events, stratified by race or region. Based on the available information on race/ethnicity or regions in the selected trials, we stratified participants into four groups: White (Caucasian), Asian, Black (African, African-American), and other participants (comprised mostly of Hispanics and Pacific Islanders). Quality assessment was made by using the Cochrane risk of bias tool. We addressed discrepancies by inviting a third author to join the discussion, and resolved disagreements by consensus.

\section{Data Synthesis and Analysis}

In each pre-specified race or region, results were reported by using the pooled odds ratio (OR) along with the $95 \%$ confidence interval $(95 \% \mathrm{Cl})$ of the composite cardiovascular events between the active antidiabetes agent group and the control group (including placebo control and active agent control). Statistical heterogeneity between trials was assessed by $R$ statistic. Fixed-effects models or randomeffects models were used according to the level of heterogeneity. Funnel plot was used to examine the 
publication bias. We performed sensitivity analyses, based on placebo use in the control group; restriction of cardiovascular outcomes to MACE; or the type of antidiabetic medication used in the active treatment groups. Statistical analyses were performed with Review Manager statistical software package (Version 5.3, Nordic Cochrane Center, Copenhagen, Denmark).

\section{Results}

\section{Included studies}

Through the search, we identified 678 records. As Fig. 1 showed, finally 16 studies were included in the quantitative synthesis. Of the included studies, 14 were placebo-controlled and 2 studies compared the test medications against active agents (Table S1). The primary outcomes of 14 placebo-controlled studies were MACE; of the other two studies, the primary outcome was hospitalization for heart failure in one and renal events in the other. The antidiabetes medications utilized in the trials included glucagonlike peptide-1 receptor analog (GLP-1RA) in 6 studies, sodium glucose cotransporter 2 inhibitors (SGLT2i) in 4 studies, and dipeptidyl-peptidase-4 (DPP-4) inhibitors in 3 studies. We included one study each that tested basal insulin, a peroxisome proliferation activated receptor (PPAR)- $y$ agonist, or an alpha glucosidase inhibitors (AGI) (Table S1).

Some notable CVOTs could not be added to our list of selected studies because they lacked data on racial/ethnic breakdown of pertinent outcomes in treatment vs. control arms. The latter included ELIXA (23), CANVAS (24), ORIGIN (25), PROactive (26) and omarigliptin cardiovascular safety trial (27).

\section{Quality assessment}

The studies all utilized a randomized, placebo- or active-controlled, double-blind, parallel treatment design. The eligibility criteria were clearly described, as were baseline characteristics in participants in the comparison groups. Overall, the risks of bias were evaluated low in most of the trials (Table S2). The visual inspection of the funnel plots indicated an even distribution of the variables (Figure S1-3).

\section{Risk of composite cardiovascular outcomes stratified by race/ethnicity}

Based on the reported event rates by race/ethnicity in the active arm and the placebo/control arm of the studies included in the present meta-analysis, there were no significant differences in the overall rates of composite cardiovascular outcomes across racial/ethnic groups (Fig. 2A-2D). However, when comparing the effects of treatment vs. control, we observed significant racial/ethnic disparities in cardiovascular outcomes of antidiabetes medication.

Among White participants, active antidiabetes medications, compared with control treatment, significantly decreased the composite cardiovascular outcomes $(\mathrm{OR}=0.90,95 \% \mathrm{Cl} 0.86-0.94, \mathrm{p}<0.05)$ (Fig. 2A). Among Asian participants, active antidiabetes medications, compared with control treatment, also significantly decreased the composite cardiovascular outcomes $(\mathrm{OR}=0.82,95 \% \mathrm{Cl} 0.76-0.90, \mathrm{p}<$ 
0.05) (Fig. 2B). However, among Black participants, active antidiabetes medications, compared with control treatment, did not significantly decrease the composite cardiovascular outcomes $(\mathrm{OR}=0.96$, $95 \% \mathrm{Cl} 0.69-1.32, \mathrm{p}=0.79$ ) (Fig. 2C). A similar pattern was found when analyzing the effects of antidiabetes medications vs. control treatment in other racial/ethnic groups comprising mostly Hispanics and Pacific Islanders (OR $=0.92,95 \% \mathrm{Cl} 0.81-1.04, \mathrm{p}=0.16$ ) (Fig. 2D). In further exploration, we combined Blacks and other groups to constitute a third block of non-White, non-Asian participants and repeated our calculations. The results in this expanded population still did not show significant reduction of composite cardiovascular outcomes in Blacks and other groups (OR 0.94; 95\% Cl 0.85-1.04) (Table 1). 
Table 1

Primary Outcomes of CVOTs Stratified by Race/Ethnicity, Combining Blacks and Other Groups*

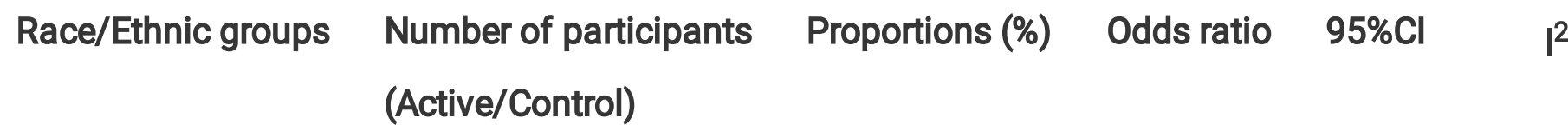

Total

\begin{tabular}{|llllll} 
White & $40396 / 40209$ & 67.15 & 0.90 & $0.86-0.94$ & $41 \%$ \\
\hline Asian & $11050 / 10643$ & 18.07 & 0.82 & $0.76-0.90$ & $46 \%$ \\
\hline Black/Others & $8862 / 8871$ & 14.78 & 0.94 & $0.85-1.04$ & $47 \%$ \\
\hline
\end{tabular}

Primary outcomes of CVOTs in placebo-controlled trials

\begin{tabular}{|llllll}
\hline White & $35288 / 35135$ & 65.96 & 0.89 & $0.85-0.93$ & $46 \%$ \\
\hline Asian & $10202 / 9781$ & 18.72 & 0.83 & $0.76-0.91$ & $44 \%$ \\
\hline Black/Others & $8169 / 8187$ & 15.32 & 0.96 & $0.86-1.06$ & $47 \%$ \\
\hline
\end{tabular}

Primary outcome restricted to MACE

\begin{tabular}{llllll} 
White & $37247 / 27094$ & 63.77 & 0.92 & $0.88-0.96$ & $8 \%$ \\
\hline Asian & $10073 / 9627$ & 19.53 & 0.86 & $0.78-0.94$ & $41 \%$ \\
\hline Black/Others & $8413 / 8432$ & 16.70 & 0.94 & $0.80-1.11$ & $51 \%$
\end{tabular}

Primary outcome in treatment with GLP-1RA

\begin{tabular}{llllll} 
White & $18203 / 18239$ & 72.98 & 0.88 & $0.82-0.94$ & $34 \%$ \\
\hline Asian & $2101 / 2130$ & 8.47 & 0.66 & $0.53-0.82$ & $0 \%$ \\
\hline Black/Others & $4637 / 4621$ & 18.54 & 0.88 & $0.77-1.01$ & $22 \%$
\end{tabular}

Primary outcome in treatment with DPP4 inhibitor

\begin{tabular}{llllll} 
White & $7010 / 6902$ & 66.87 & 0.97 & $0.88-1.07$ & $0 \%$ \\
\hline Asian & $1342 / 1369$ & 13.03 & 1.03 & $0.80-1.31$ & $0 \%$ \\
\hline Black/Others & $2067 / 2115$ & 20.10 & 1.30 & $1.07-1.58$ & $0 \%$
\end{tabular}

Primary outcome in treatment with SGLT2 inhibitor

\begin{tabular}{llllll} 
White & $9992 / 9925$ & 70.18 & 0.84 & $0.77-0.91$ & $71 \%$ \\
\hline Asian & $3076 / 2620$ & 20.07 & 0.72 & $0.60-0.85$ & $30 \%$ \\
\hline Black/Others & $1395 / 1370$ & 9.75 & 0.79 & $0.61-1.03$ & $0 \%$
\end{tabular}

Primary outcome in treatment with basal insulin

*Hispanics, Pacific Islanders, Hawaiian, American Indian, Alaska Native 


\begin{tabular}{|c|c|c|c|c|c|}
\hline Race/Ethnic groups & $\begin{array}{l}\text { Number of participants } \\
\text { (Active/Control) }\end{array}$ & Proportions (\%) & Odds ratio & $95 \% \mathrm{Cl}$ & $1^{2}$ \\
\hline White & $2891 / 2882$ & 79.51 & 0.96 & $0.80-1.14$ & / \\
\hline Asian & $317 / 332$ & 8.94 & 0.42 & $0.21-0.81$ & / \\
\hline Black/Others & $432 / 407$ & 11.55 & 0.56 & $0.34-0.95$ & / \\
\hline \multicolumn{6}{|c|}{ Primary outcome in treatment with PPAR antagonist } \\
\hline White & $2300 / 2259$ & 63.92 & 1.03 & $0.85-1.25$ & / \\
\hline Asian & $942 / 942$ & 26.42 & 0.72 & $0.50-1.03$ & / \\
\hline Black/Others & $331 / 358$ & 9.66 & 0.87 & $0.53-1.43$ & / \\
\hline \multicolumn{6}{|c|}{ Primary outcome in treatment with AGI } \\
\hline White & / & 0 & / & / & / \\
\hline Asian & $3272 / 3250$ & 100 & 0.97 & $0.85-1.11$ & / \\
\hline Black/Others & / & 0 & / & / & / \\
\hline
\end{tabular}

\section{Sensitivity analyses}

We performed sensitivity analyses based on assessment of only placebo-controlled studies, restriction of outcomes to MACE, and type of antidiabetes medication. Evaluation of only the placebo-controlled trials did not change our main findings: the reduction in composite cardiovascular outcomes was significant in White and Asian patients, but not in Blacks or other groups (Table S3 and Figure S4-S7).

The overall pattern observed for the primary outcomes was recapitulated when analysis was restricted to MACE without considering other outcomes. Significant reductions in MACE occurred in White and Asian patients, but not in Black patients or participants from other racial/ethnic groups (Table S3 and Figure S8S11).

There appeared to be racial/ethnic differences in the cardiovascular effects of certain medications. CVOTs that utilized GLP-1RA as treatment demonstrated significant decrease of the composite cardiovascular outcomes in both White and Asian patients, but not in Blacks or other groups (Table S3 and Fig. 2A-2D). When compared with the control group, treatment with SGLT2 inhibitors resulted in point estimates for risk reduction that were nominally greater in Asians (OR 0.72) and Blacks (OR 0.73) compared with Whites (OR 0.84) and other groups (OR 0.82). However, owing to wide confidence intervals, the effects were significant only in White and Asian patients (Table S3 and Fig. 2A-2D). With DPP-4 inhibitors, there was a neutral effect in Whites and an apparent trend toward increased composite 
cardiovascular outcomes in non-White groups that reached statistical significance in Black patients (OR $=1.36,95 \% \mathrm{Cl} 1.04-1.77, \mathrm{p}<0.05)$ (Table S3 and Fig. 2A-2D).

The only trial of basal insulin included in the present meta-analysis compared degludec treatment with an active control agent (glargine), and reported a significant reduction in composite cardiovascular outcomes in both Black patients (OR 0.29; $95 \% \mathrm{Cl} 0.11-0.75)$ and Asian patients (OR 0.42; $95 \% \mathrm{Cl} 0.21-$ 0.81 ), but not in White or in other groups (Table S3 and Fig. 2A-2D). There was incomplete racial/ethnic information regarding cardiovascular outcomes of treatment with PPAR-y antagonist and AGI, but their effects were generally neutral in the populations studied (Table S3 and Fig. 2A-2D).

\section{Discussion}

Our meta-analysis of 16 RCTs of antidiabetes medications that pre-specified cardiovascular outcomes and provided adequate pertinent data showed that the overall event rates did not differ significantly among participants from the different racial/ethnic groups. The latter finding probably reflects the inclusion criteria that targeted high-risk patients with type 2 diabetes for enrollment in the CVOTs.

However, when comparing the effects of antidiabetes medication versus placebo- or active-control treatment by racial/ethnic group, we did observe disparities in the magnitude and significance of cardiovascular risk reduction. In general, a significant reduction in the composite cardiovascular outcomes was achieved in Asian and White participants, but not in Blacks and other racial/ethnic groups (including Hispanics and Pacific Islanders).

Sensitivity analyses showed consistency of our main findings of racial/ethnic disparities in cardiovascular risk reduction across the two antidiabetes medication classes (GLP1-RA and SGLT2 inhibitors) that have demonstrated superiority over placebo in CVOTs. However, the findings regarding other agents that have not been reported to decrease cardiovascular risk (DPP-4 inhibitors, PPAR- $\gamma$ agonist, basal insulin) were discordant. We observed a neutral effect of PPAR-y agonist on composite cardiovascular outcomes, without racial/ethnic disparities; a neutral effect in Whites and Asians, but an apparent increased risk among Blacks and other groups in trials of DPP-4 inhibitors; and a neutral effect in Whites, but significantly decreased risk in Asians, Blacks and other groups in the trial of basal insulin. Thus, our overall meta-analysis results were strongly influenced by data from the studies that tested the effects of GLP1-RA and SGLT2 inhibitors on cardiovascular outcomes.

Given that the individual studies in our meta-analysis were powered for the overall enrolled population, not racial sub-groups, our present findings of disparities in cardiovascular outcomes must be taken with caution. Nonetheless, the consistent pattern of lack of significant reduction in cardiovascular outcomes with antidiabetes medications among Blacks and other non-White, non-Asian participants enrolled in the CVOTs is striking. The reduction of cardiovascular events requires interventions to control several comorbid risk factors beyond glycemia. Optimal control of hypertension, dyslipidemia, inflammation, albuminuria; effective counseling regarding smoking cessation, are all components of a comprehensive strategy for maintaining good cardiovascular health $(14,15,18)$. 
The CVOTs included in the present meta-analysis generally focused on executing a specific study protocol, leaving the management of the numerous co-morbid risk factors to the discretion of community health care providers. Thus, it is plausible that heterogeneity in access to care and quality of control of co-morbid risk factors could have contributed to our findings of racial/ethnic disparities in the reduction of composite cardiovascular outcomes $(9-11,16,28,29)$. Indeed, there is ample documentation of the unevenness of cardiovascular risk factors across demographic groups; notably, higher rates of hypertension, albuminuria, and chronic kidney disease have been reported in Black, Latino and Native American populations compared with Whites $(18,30)$. The combination of diabetes and kidney disease increases the risk of cardiovascular mortality 3-fold compared with diabetes without kidney disease (31).

Although the overall rates of cardiovascular outcomes were similar among participants from various racial/ethnic groups in the CVOTs, perhaps reflecting the inclusion criteria that targeted high-risk patients with type 2 diabetes, the failure to demonstrate consistent risk reduction across groups requires further scrutiny. Studies that enrolled multi-ethnic participants have shown that, with equal access to care and equal treatment, disparities in clinical outcomes (including mortality) can be ameliorated $(12,13,29,32$, 33).

Like all meta-analyses, the present study has some limitations. The capture of cardiovascular events by race/ethnicity was incomplete, as several notable CVOTs (23-27) did not report a breakdown of MACE by race/ethnicity in the treatment and control groups. Thus, despite our best efforts to be comprehensive and inclusive, the lack of publicly available data on pertinent racial/ethnic results forced us to rely mainly on CVOTs that used GLP-1RAs, DPP-4 inhibitors, SGLT2 inhibitors and basal insulin, which introduces possible selection bias. Also, although the level of heterogeneity across studies was low in this metaanalysis, data from separate studies with varied baseline characteristics, treatment regimen, and duration of follow-up were combined for analysis. Thus, our results should be interpreted with caution. Furthermore, the studies we examined seldom provided detailed information on baseline cardiovascular risk factors by race/ethnicity, which precluded further meta-regression analysis of possible explanatory variables related to our finding of disparities in cardiovascular outcomes.

Finally, and most importantly, the sample from Black and other racial/ethnic groups (Hispanics, Native Americans, Native Hawaiians, Pacific Islanders and residents of Oceania, Latin America and the Caribbean) was relatively small, which might have lowered statistical power to detect differences. Data from the U.S. Census Bureau indicate that, of the estimated 326 million total population, $60.6 \%$ selfidentified as non-Hispanic Whites, compared with 18.3\% Hispanics, $12.3 \%$ non-Hispanic Blacks, 5.6\% Asians and $0.9 \%$ American Indians (34). The United Nations estimates that there are 1.34 billion people living in Africa (17.2\% of the world's population (35). Thus, the proportion of Blacks (4.3\%) in our total meta-analysis population of 120,031 participants enrolled in the CVOTs indicates a marked underrepresentation of the Black population in the U.S. or globally. The enrollment of Hispanics in the CVOTs $(<10 \%)$ also is low relative to the $18.3 \%$ Hispanic representation in the U.S. population, but fairly comparable to the Latin America and the Caribbean representation (8.3\%) in the global population (35). 
In conclusion, the present meta-analysis shows that the aggregate point estimates and confidence intervals for the numerous agents that have been tested in CVOTs reached statistical significance for reduction of composite cardiovascular outcomes only in White and Asian participants, who together represented $85.2 \%$ of the 120,031 participants enrolled in those trials. For Blacks and other racial/ethnic groups (who together represented $14.8 \%$ of the enrollment), the meta-analysis showed general cardiovascular safety but not significant reduction in composite cardiovascular outcomes by the medications tested in the CVOTs. Given the disproportionate burden of type 2 diabetes and its complications in African Americans, Latinos, Native Americans and other ethnic groups, it is imperative to demonstrate the efficacy of medications and interventions for cardiovascular risk reduction in these populations. The suboptimal representation of Blacks and other high-risk groups in the populations enrolled in CVOTs leaves open the question as to whether the benefits demonstrated for Whites and Asians in these studies can be expected to apply to other groups. Clearly, adequately powered studies are required to answer this important public health question.

\section{Declarations}

\section{Ethics approval and consent to participate}

No applicable.

\section{Consent for publication}

No applicable.

\section{Availability of data and materials}

Data of all the clinical trials included in this meta-analysis could be reached from the published manuscripts.

\section{Competing interests}

Linong Ji has received fees for lecture presentations and consulting fees from AstraZeneca, Merck, Novartis, Novo Nordisk, Lilly, Roche, Sanofi-Aventis and Takeda, and grants/research support from AstraZeneca, Merck, Novartis, Novo Nordisk and Sanofi-Aventis; S.D.-J. has led clinical trials for AstraZeneca, Novo Nordisk, Inc., and Boehringer Ingelheim; has received consulting fees from AstraZeneca, Boehringer Ingelheim, Janssen, Merck \& Co. Inc., and Sanofi; and has equity interests in Jana Care, Inc. and Aerami Therapeutics; no other relationships or activities that could appear to have influenced the submitted work.

\section{Funding}

This study was supported by National Natural Science Foundation of China (No.81970708 and No.81970698) and was also funded by Lilly China company. SD-J is supported, in part, by a grant from 
the National Institutes of Health (R01 DK067269). The funding sources had no role in the study design, data collection or analysis, decision to publish, or preparation of the manuscript.

\section{Authors' contributions}

LJ and XC conceived and designed the study. XC and CL assisted with the methods. XC, CL and WY did the data analysis. XC and LJ drafted the initial manuscript. SD-J discussed study concept, reviewed and revised manuscript. All authors assisted with interpretation, commented on drafts of the manuscript, and approved the final version. LJ is the guarantor and attests that all listed authors meet authorship criteria and that no others meeting the criteria have been omitted.

\section{Acknowledgements}

We thank doctors, nurses and technicians for their practical work during the study at Department of Endocrinology and Metabolism in Peking University People's Hospital.

\section{Abbreviations}

AGI, alpha glucosidase inhibitor; CENTRAL, the Cochrane Central Register of Controlled Trials; CHD, coronary heart disease; $\mathrm{Cl}$, confidence interval; CVD, cardiovascular disease; DPP-4, dipeptidyl-peptidase4; GLP-1RA, glucagon-like peptide-1 receptor analog; MACE, major adverse cardiovascular events; NHANES, National Health and Nutrition Examination Survey; OR, odd ratio; PPAR, peroxisome proliferation activated receptor; RCT, randomized control trial; SGLT2i, sodium glucose cotransporter 2 inhibitor; T2DM, type 2 diabetes mellitus.

\section{References}

1. Centers for Disease Control and Prevention. National diabetes statistics report. 2017: estimates of diabetes and its burden in the United States. Available at: https://www.cdc.gov/diabetes/pdfs/data/statistics/national-diabetes-statistics-report.pdf. (accessed April30, 2020).

2. Agbim U, Carr RM, Pickett-Blakely O, et al. Ethnic disparities in adiposity: Focus on non-alcoholic fatty liver disease, visceral, and generalized obesity. Curr Obes Rep. 2019;8(3):243-54.

3. Rodríguez JE, Campbell KM. Racial and Ethnic Disparities in prevalence and care of patients with type 2 diabetes. Clin Diabetes. 2017;35(1):66.

4. Egede LE, Dagogo-Jack S. Epidemiology of Type 2 Diabetes: Focus on Ethnic Minorities. Med Clin N Am. 2005;89:949-75.

5. Dagogo-Jack S. Ethnic disparities in type 2 diabetes: Pathophysiology and implications for prevention and management. J Natl Med Assoc. 2003;(95):774-789. 
6. Cowie CC, Rust KF, Byrd-Holt DD, et al. Prevalence of diabetes and impaired fasting glucose in adults in the U.S. population: National Health And Nutrition Examination Survey 1999-2002. Diabetes Care. 2006;29:1263-68.

7. Cowie CC, Rust KF, Byrd-Holt DD, et al. Prevalence of diabetes and high risk for diabetes using A1C criteria in the U.S. population in 1988-2006. Diabetes Care. 2010;33:562-68.

8. Tillin T, Hughes AD, Mayet $J$, et al. The relationship between metabolic risk factors and incident cardiovascular disease in Europeans, South Asians, and African Caribbeans: SABRE (Southall and Brent Revisited): A prospective population-based study. J Am Coll Cardiol. 2013;61:1777-86.

9. Ramachandran A, Ma RC, Snehalatha C. Diabetes in Asia. Lancet. 2010;375:408-18.

10. Canedo JR, Miller ST, Schlundt D, et al. Racial/Ethnic disparities in diabetes quality of care: the role of healthcare access and socioeconomic status. J Racial Ethn Health Disparities. 2018;5(1):7-14.

11. Dagogo-Jack S, Funnell M, Davidson J. Barriers to achieving optimal glycemic control in a multiethnic society. Current Diabetes Reviews. 2006;2:285-93.

12. Dagogo-Jack S. Preventing diabetes-related morbidity and mortality in the primary care setting. J Natl Med Assoc. 2002;94:549-60.

13. Karter AJ, Ferrara A, Liu JY, et al. Ethnic disparities in diabetic complications in an insured population. JAMA. 2002;287(19):2519-27.

14. Gaede $\mathrm{P}$, Lund-Andersen $\mathrm{H}$, Parving $\mathrm{HH}$, et al. Effect of a multifactorial intervention on mortality in type 2 diabetes. N Engl J Med. 2008;358:580.

15. Ridker PM, Danielson E, Fonseca FA, et al. JUPITER Study Group. Rosuvastatin to prevent vascular events in men and women with elevated C-reactive protein. N Engl J Med. 2008;359(21):2195-207.

16. Rana JS, Liu JY, Moffet HH, et al. Ethnic Differences in Risk of Coronary Heart Disease in a Large Contemporary Population. Am J Prev Med. 2016;50(5):637-41.

17. Ford ES, Giles WH, Dietz W. Prevalence of the metabolic syndrome among US adults, findings from the Third National Health and Nutrition Examination Survey. JAMA. 2002;287:356.

18. Emelia J, Benjamin P, Muntner A, Alonso, et al. Heart Disease and Stroke Statistics-2019 Update: A Report From the American Heart Association. Circulation. 2019;139:e56-28.

19. Dagogo-Jack I, Dagogo-Jack S. Dissociation between cardiovascular risk markers and clinical outcomes in African Americans: Need for greater mechanistic insight. Current Cardiovascular Risk Reports. 2011;5:200-6.

20. Action to Control Cardiovascular Risk in Diabetes Study Group. Effects of intensive glucose lowering in type 2 diabetes. N Engl J Med. 2008;358:2545.

21. ADVANCE Collaborative Group. Intensive blood glucose control and vascular outcomes in patients with type 2 diabetes. N Engl J Med. 2008;358:2560.

22. Duckworth W, Abraira C, Moritz T, et al. VADT Investigators. Glucose control and vascular complications in veterans with type 2 diabetes. N Engl J Med. 2009;360:129. 
23. Pfeffer MA, Claggett B, Diaz R, et al. Lixisenatide in Patients with Type 2 Diabetes and Acute Coronary Syndrome. N Engl J Med. 2015;373:2247-57.

24. Neal B, Perkovic V, Mahaffey KW, et al. Canagliflozin and Cardiovascular and Renal Events in Type 2 Diabetes. N Engl J Med. 2017;377(7):644-57.

25. Gerstein HC, Bosch J, Dagenais GR, et al. Basal insulin and cardiovascular and other outcomes in dysglycemia. N Engl J Med. 2012;367:319-28.

26. Dormandy JA, Charbonnel B, Eckland DJ, et al. Secondary prevention of macrovascular events in patients with type 2 diabetes in the PROactive Study (PROspective pioglitAzone Clinical Trial In macroVascular Events): a randomised controlled trial. Lancet. 2005;366:1279-89.

27. Gantz I, Chen M, Suryawanshi S,et al. A randomized, placebo-controlled study of the cardiovascular safety of the once-weekly DPP-4 inhibitor omarigliptin in patients with type 2 diabetes mellitus. Cardiovasc Diabetol. 2017;16(1):112.

28. Harris MI. Racial and ethnic differences in health care access and health outcomes for adults with type 2 diabetes. Diabetes Care. 2001;24:454-59.

29. Cai A, Dillon C, Hillegass WB, et al. Risk of Major adverse cardiovascular events and major hemorrhage among white and black patients undergoing percutaneous coronary intervention. $\mathrm{J}$ Am Heart Assoc. 2019;8(22):e012874.

30. Norris KC, Agodoa LY. Unraveling the racial disparities associated with kidney disease. Kidney Int. 2005;68(3):914-24.

31. Afkarian M, Sachs MC, Kestenbaum B, et al. Kidney disease and increased mortality risk in type 2 diabetes. J Am Soc Nephrol. 2013;24(2):302-8.

32. Conway BN, May ME, Blot WJ. Mortality Among Low-Income African Americans and Whites With Diabetes. Diabetes Care. 2012;35(11):2293-9.

33. Knowler WC, Barrett-Connor E, Fowler SE, et al. Reduction in the incidence of type 2 diabetes with lifestyle intervention or metformin. N Engl J Med. 2002;346(6):393-403.

34. U.S. Census Bureau. United States of America: Race and Ethnicity. https://data.census.gov/cedsci/profile? $q=$ United\%20States\&g=0100000US\&tid=ACSDP1Y2018.DP05 (accessed April 27, 2020).

35. United Nations

Department of Economic and Social Affairs Population Dynamics United Nations. Department of Economic and Social Affairs Population Dynamics. https://population.un.org/wpp/Download/Standard/Population/(accessed April 27, 2020).

\section{Figures}


678 record identified through database searching

in Pubmed, Medline, Embase and the Cochrane

Center Register of Controlled Trials for Studies

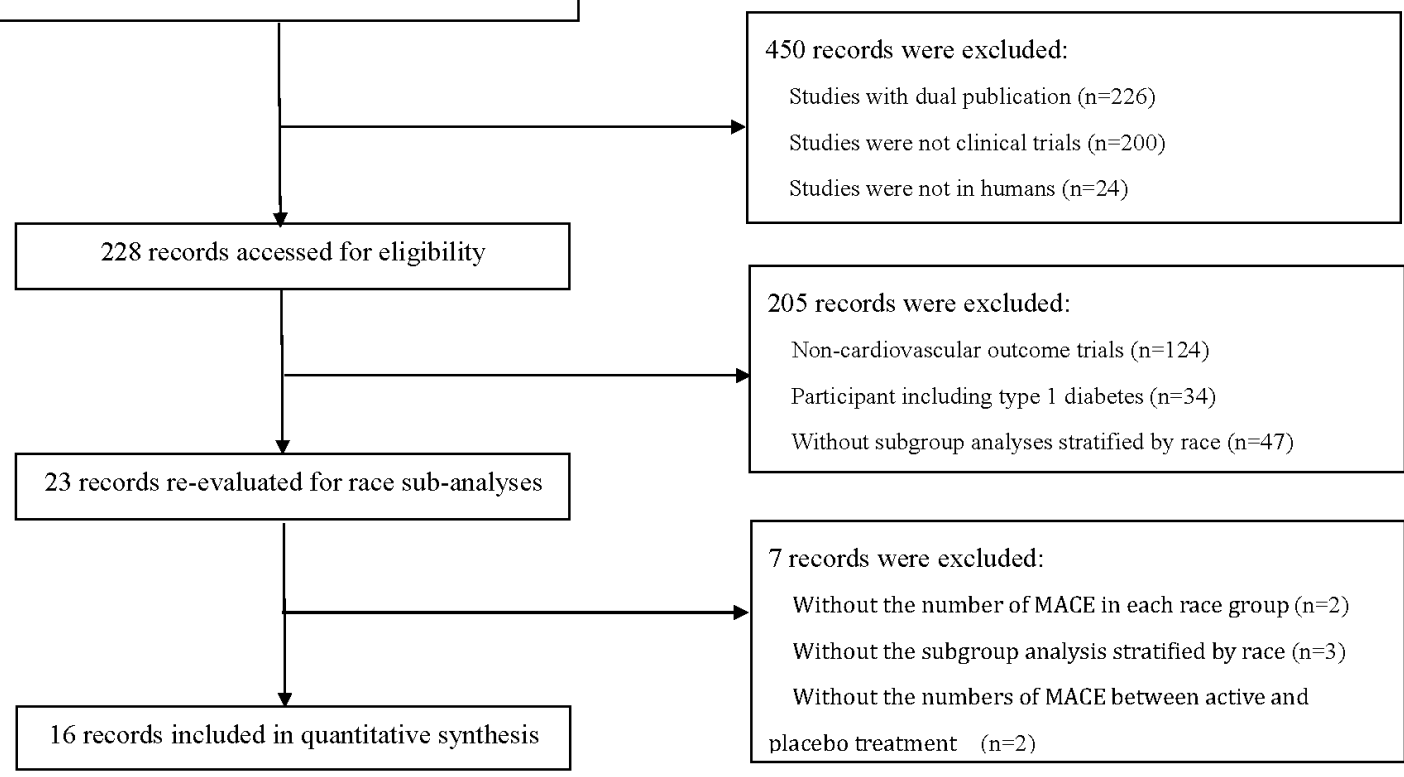

Figure 1

Flowchart of search results 
A

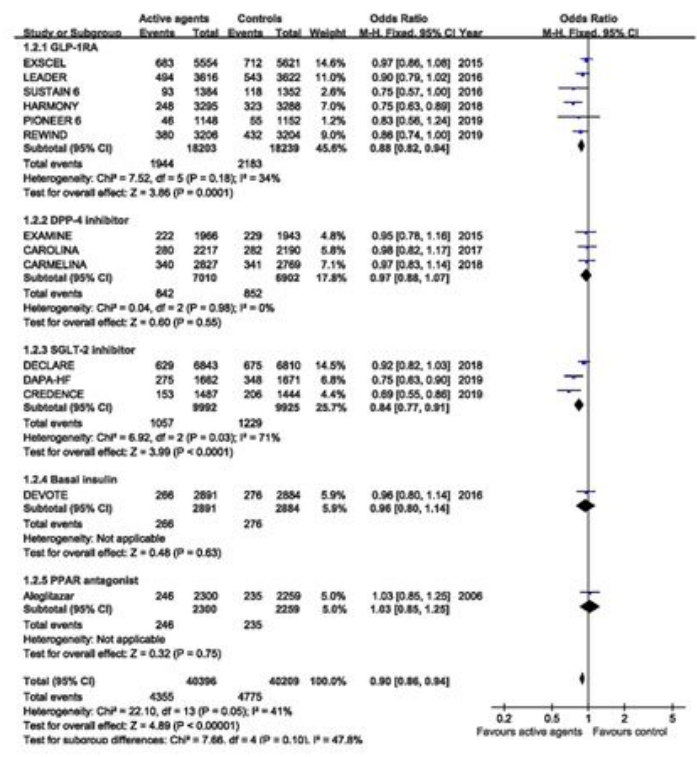

C

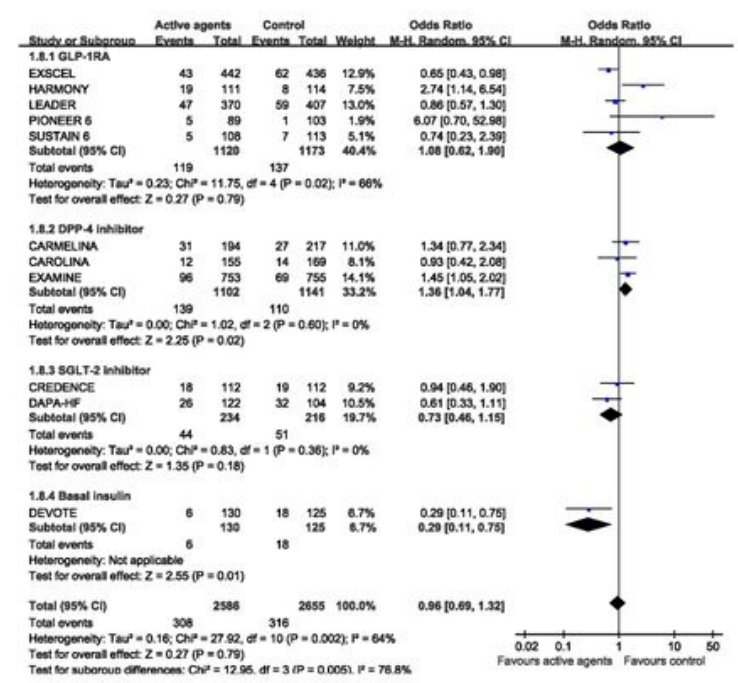

B

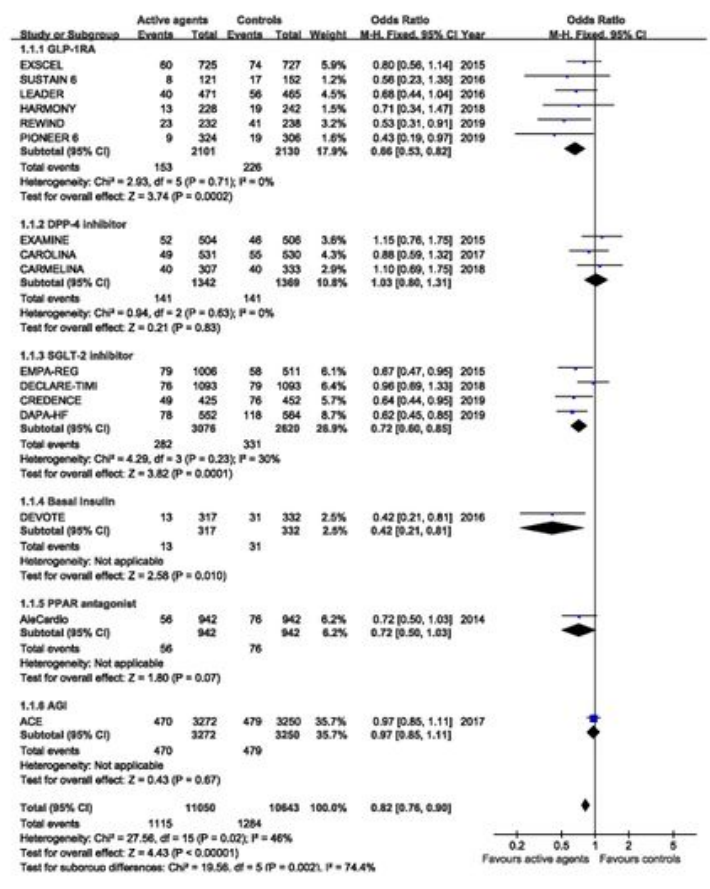

$\mathrm{D}$

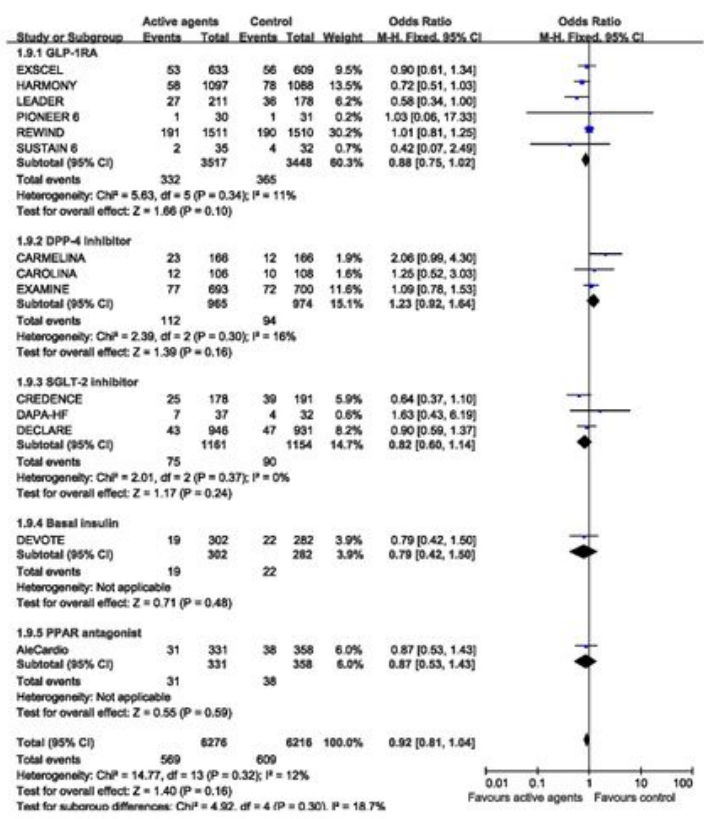

\section{Figure 2}

Primary outcomes of CVOTs in White patients, Asian patients, black patients and patients with type 2 diabetes from other racial/ethnic groups* Figure 2A: Primary outcomes of CVOTs in White patients with type 2 diabetes; Figure 2B: Primary outcomes of CVOTs in Asian patients with type 2 diabetes; Figure 2C: Primary outcomes of CVOTs in black patients with type 2 diabetes; Figure 2D: Primary outcomes of 
CVOTs in patients with type 2 diabetes from other racial/ethnic groups* *Hispanics, Pacific Islanders, Hawaiian, American Indian, Alaska Native

\section{Supplementary Files}

This is a list of supplementary files associated with this preprint. Click to download.

- RacialdifferenceinCVOTsSupplementaryMaterials.docx 